\title{
PARABOLIC BESOV REGULARITY FOR THE HEAT EQUATION
}

\author{
HUGO AIMAR AND IVANA GÓMEZ
}

\begin{abstract}
We obtain parabolic Besov smoothness improvement for temperatures on cylindrical regions based on Lipschitz domains. The results extend those for harmonic functions obtained by S. Dahlke and R. DeVore in Besov regularity for elliptic boundary value problems published in Communications in Partial Differential Equations 22, no. 1-2, 1-16, 1997.
\end{abstract}

\section{INTRODUCTION}

S. Dahlke and R. DeVore prove in [DD] that a particular imbedding property in the scale of Besov spaces $B_{p, q}^{\alpha}(D)$ when $q=p\left(B_{p, p}^{\alpha}(D)=: B_{p}^{\alpha}(D)\right)$ holds when we restrict our attention to harmonic functions on a Lipschitz domain $D$ of $\mathbb{R}^{d}$. Precisely in Theorem 3.2 in [DD] the authors prove that if $D$ is a Lipschitz domain in $\mathbb{R}^{d}, 1<p<\infty, \lambda>0,0<\alpha<\frac{\lambda d}{d-1}$ and $\frac{1}{\tau}=\frac{1}{p}+\frac{\alpha}{d}$, then

$$
\mathscr{H}(D) \cap B_{p}^{\lambda}(D) \subset B_{\tau}^{\alpha}(D),
$$

where $\mathscr{H}(D)$ is the space of all harmonic functions defined on $D$.

Since we can choose the parameter $\alpha$ to be larger than $\lambda$, the result is relevant in order to improve the rate of convergence of nonlinear methods of approximation. For details, regarding this application of (1.1) we refer to [DD].

In this paper we aim to prove similar results for temperatures. We shall write $\Omega$ to denote the parabolic cylinder $D \times(0, T)$ with $T>0$ and $D$ a bounded Lipschitz domain in $\mathbb{R}^{d}(d \geq 2)$. Let $\Theta(\Omega)$ denote the space of all temperatures $u=u(x, t)$ in $\Omega$. In other words $\Theta(\Omega)=\left\{u: \frac{\partial u}{\partial t}=\Delta u\right.$ in $\left.\Omega\right\}$.

Let us briefly introduce the parabolic Besov scale $\mathbb{B}_{r}^{\gamma}(\Omega)$ for $0<\gamma<1,1<r<$ $\infty$. Among the several approaches to Besov spaces, we choose the interpolation one. For $0<\gamma<1,1<r, s<\infty$ define

$$
B_{r, s}^{\gamma, \frac{\gamma}{2}}(\Omega)=\left(L_{r}(\Omega), W_{r}^{2,1}(\Omega)\right)_{\frac{\gamma}{2}, s}
$$

and $\mathbb{B}_{r}^{\gamma}(\Omega)=B_{r, r}^{\gamma, \frac{\gamma}{2}}(\Omega)$. Here $W_{r}^{2,1}(\Omega)$ is the anisotropic Sobolev space defined by the norm

$$
\|v\|_{W_{r}^{2,1}(\Omega)}=\|v\|_{L_{r}(\Omega)}+\sum_{i=1}^{d}\left\|\frac{\partial v}{\partial x_{i}}\right\|_{L_{r}(\Omega)}+\sum_{i=1}^{d} \sum_{j=1}^{d}\left\|\frac{\partial^{2} v}{\partial x_{i} \partial x_{j}}\right\|_{L_{r}(\Omega)}+\left\|\frac{\partial v}{\partial t}\right\|_{L_{r}(\Omega)} .
$$

2010 Mathematics Subject Classification. Primary 35B65, 35K05.

Key words and phrases. Solutions of the heat equation, Besov regularity.

The research was supported by CONICET, UNL and ANPCyT. 
For general $\gamma>0$ we can define $\mathbb{B}_{r}^{\gamma}(\Omega)$ through the action of the derivatives $\frac{\partial}{\partial t}$ and $\frac{\partial^{2}}{\partial x_{i} \partial x_{j}}$.

We are now in position to state our results which give improvements of Besov regularity for temperatures in two different directions.

Theorem 1. Let $1<p<\infty, \lambda>0$, $\ell$ the largest integer less than $\lambda+d, 0<\alpha<$ $\min \left\{\ell, \frac{\lambda d}{d-1}\right\}$ and $\frac{1}{\tau}=\frac{1}{p}+\frac{\alpha}{d}$. Then

$$
\Theta(\Omega) \cap L_{p}\left((0, T) ; B_{p}^{\lambda}(D)\right) \subset L_{\tau}\left((0, T) ; B_{\tau}^{\alpha}(D)\right) .
$$

Theorem 2. Let $1<p<\infty, \lambda>0,0<\alpha<\min \left\{d\left(1-\frac{1}{p}\right), \frac{\lambda d}{d-1}\right\}$ and $\frac{1}{\tau}=\frac{1}{p}+\frac{\alpha}{d}$. Then

$$
\Theta(\Omega) \cap \mathbb{B}_{p}^{\lambda}(\Omega) \subset \bigcap_{\alpha>\varepsilon>0} \mathbb{B}_{\tau}^{\alpha-\varepsilon}(\Omega) .
$$

Theorem 2 provides the analogous for temperatures of (1.1).

As in the elliptic case a central tool for our results is the use of the characterization of the elliptic Besov spaces through Daubechies wavelet bases. For our first result we shall use the parabolic analogous of Theorem 3.1 in [DD], essentially contained in Corollary 5.2 of [AGI1]. Our second result is a corollary of the first one if we use Theorem 1.1 in [AGI2].

In $\S 2$ we introduce the spaces involved in theorems 1 and 2 and the wavelet characterization of the elliptic Besov spaces. Section 3 contains estimates for the space gradients of temperatures which are essentially proved in [AGI1]. In Section 4 we introduce partitions of the wavelet index set at each scale and each time and we prove some central technical lemmas. Finally Section 5 is devoted to prove theorems 1 and 2 .

\section{BESOV SPACES}

The literature on classical Besov spaces and their extensions to parabolic settings is abundant, see [BIN], [Pee], [Sch], [ST], [Bow]. Let $D$ be a bounded Lipschitz domain in $\mathbb{R}^{d}$. Under this regularity condition on $\partial D$ we have that the Besov space $B_{r}^{\gamma}(D)$ coincides with the space of the restrictions to $D$ of $B_{r}^{\gamma}\left(\mathbb{R}^{d}\right)$. A basic tool in [DD] which shall also be used here in the proof of Theorem 1 , is the characterization of $B_{r}^{\gamma}\left(\mathbb{R}^{d}\right)$ in terms of Daubechies type wavelets. We shall closely follow, with minor changes, the notation in [DD] in order to state the characterization results that we shall use in the sequel. Let $\mathcal{D}_{j}, j \in \mathbb{Z}$, be the set of all dyadic cubes in $\mathbb{R}^{d}$ of measure $2^{-j d}$. Set $\mathcal{D}=\cup_{j \in \mathbb{Z}} \mathcal{D}_{j}$ to denote the family of all dyadic cubes in $\mathbb{R}^{d}$ and $\mathcal{D}^{+}$to denote those cubes in $\mathcal{D}$ with measure less than or equal to 1.

For any given positive integer $n$ there exists a set $\Psi$ of $2^{d}-1$ functions $\psi \in$ $\mathscr{C}_{0}^{n}\left(\mathbb{R}^{d}\right)$, with $n$-vanishing moments and $\phi \in \mathscr{C}_{0}^{n}\left(\mathbb{R}^{d}\right)$ all of them supported in a cube $Q$ centered at the origin of $\mathbb{R}^{d}$, such that the system $\left\{\psi_{I}: \psi \in \Psi, I \in \mathcal{D}\right\}$ is an orthonormal basis for $L^{2}\left(\mathbb{R}^{d}\right)$ and $\left\{\psi_{I}: \psi \in \Psi, I \in \mathcal{D}^{+}\right\}$is an orthonormal basis for the complement of $S_{0}$, the $L^{2}\left(\mathbb{R}^{d}\right)$ closure of the linear span of $\left\{\phi_{I}: I \in \mathcal{D}_{0}\right\}$. Here, as usual, $\psi_{I}$ and $\phi_{I}$ denote the translation and scaling of $\psi$ and $\phi$ defined

by the dyadic cube $I \in \mathcal{D}, \psi_{I}(x)=2^{\frac{j d}{2}} \psi\left(2^{j} x-k\right)$ with $I=\Pi_{l=1}^{d}\left[\frac{k_{l}}{2^{j}}, \frac{k_{l}+1}{2^{j}}\right]$ and $k=\left(k_{1}, \ldots, k_{d}\right)$. We point out here that the support of each $\psi_{I}$ is contained in a cube $Q(I)$ containing $I$ with $\frac{|Q(I)|}{|I|}=|Q|$. 
Once and for ever along this paper we shall fix the restrictions of all the involved parameters. The space dimension $d$ is larger than or equal to two. The starting integrability parameter $p$ is finite and larger than one. The starting regularity parameter $\lambda$ is positive. The improved regularity parameter $\alpha$ belongs to the open interval $\left(0, \min \left\{\ell, \frac{\lambda d}{d-1}\right\}\right)$ with $\ell$ the largest integer less than $\lambda+d$. The final integrability, which is worse than the starting one, is given by the positive parameter $\tau$ defined by $\frac{1}{\tau}=\frac{1}{p}+\frac{\alpha}{d}$. Since we shall deal with these parameters when considering the elliptic Besov spaces, we will use their wavelet characterization for the integrability parameter less than one but only for positive regularity.

It will be useful for further convenience to write out explicitly the characterizations of $B_{p}^{\lambda}\left(\mathbb{R}^{d}\right)$ and $B_{\tau}^{\alpha}\left(\mathbb{R}^{d}\right)$ with the above relationships among the parameters. We shall also adopt here the $L_{p}\left(\mathbb{R}^{d}\right)$ normalization of the wavelet basis introduced in [DD]. For $\psi \in \Psi$, and $I \in \mathcal{D}$, with $\psi_{I, p}$ we denote the function

$$
\psi_{I, p}=|I|^{\frac{1}{2}-\frac{1}{p}} \psi_{I} .
$$

Notice that $\psi_{I}=\psi_{I, 2}$ and that $\left\|\psi_{I, p}\right\|_{L_{p}\left(\mathbb{R}^{d}\right)}=\|\psi\|_{L_{p}\left(\mathbb{R}^{d}\right)}$ for every $I \in \mathcal{D}$. Let us also observe that with this notation the expansion of a function $f$ in this wavelet basis is given by

$$
\begin{aligned}
f & =\sum_{I \in \mathcal{D}} \sum_{\psi \in \Psi}\left\langle f, \psi_{I}\right\rangle \psi_{I} \\
& =\sum_{I \in \mathcal{D}} \sum_{\psi \in \Psi}\left\langle f, \psi_{I, p^{\prime}}\right\rangle \psi_{I, p},
\end{aligned}
$$

where $p^{\prime}$ is the Hölder conjugate of $p$. When the wavelet decomposition is only used at the scales defined by $|I| \leq 1$ we only have the cubes in $\mathcal{D}^{+}$. And the representation of $f$ becomes now

$$
f=P_{0} f+\sum_{I \in \mathcal{D}^{+}} \sum_{\psi \in \Psi}\left\langle f, \psi_{I, p^{\prime}}\right\rangle \psi_{I, p}
$$

with $P_{0}$ the orthogonal projector on $S_{0}=\overline{\operatorname{span}\left\{\phi_{I}: I \in \mathcal{D}_{0}\right\}}$, the closure in the $L^{2}$ sense.

Proposition 3. Let $d, p, \lambda, \alpha$ and $\tau$ be as before. Assume that $\Psi \subset \mathscr{C}^{n}\left(\mathbb{R}^{d}\right)$ with $n>\lambda+d$. Then

(A) $f \in B_{p}^{\lambda}\left(\mathbb{R}^{d}\right)$ if and only if

$$
\left\|P_{0} f\right\|_{L_{p}\left(\mathbb{R}^{d}\right)}+\left(\sum_{I \in \mathcal{D}^{+}} \sum_{\psi \in \Psi}|I|^{-\frac{\lambda p}{d}}\left|\left\langle f, \psi_{I, p^{\prime}}\right\rangle\right|^{p}\right)^{\frac{1}{p}}<\infty .
$$

(B) $f \in B_{\tau}^{\alpha}\left(\mathbb{R}^{d}\right)$ if and only if

$$
\left\|P_{0} f\right\|_{L_{\tau}\left(\mathbb{R}^{d}\right)}+\left(\sum_{I \in \mathcal{D}^{+}} \sum_{\psi \in \Psi}\left|\left\langle f, \psi_{I, p^{\prime}}\right\rangle\right|^{\tau}\right)^{\frac{1}{\tau}}<\infty .
$$

For a proof see $[\mathrm{FJ}]$, [Mey].

Less standard are the parabolic Besov spaces. For our approach to the proof of Theorem 2 we shall only need the interpolation version of this scale and no wavelet 
characterization is required. For characterizations of anisotropic Besov spaces see [Lei] and [Bow]. Since the hypothesis $\alpha<d\left(1-\frac{1}{p}\right)$ in Theorem 2 guarantees that $\tau>1$ we shall only concentrate our attention to integrability exponents larger than one when dealing with this parabolic scale of Besov spaces. For $0<\gamma<1$ and $1<r, s<\infty$ define

$$
B_{r, s}^{\gamma, \frac{\gamma}{2}}(\Omega)=\left(L_{r}(\Omega), W_{r}^{2,1}(\Omega)\right)_{\frac{\gamma}{2}, s}
$$

and $\mathbb{B}_{r}^{\gamma}(\Omega)=B_{r, r}^{\gamma, \frac{\gamma}{2}}(\Omega)$. The parabolic Sobolev space $W_{r}^{2,1}(\Omega)$ is the space of those functions in $L_{r}(\Omega)$ with weak derivatives of order two in space variables and order one in time in $L_{r}(\Omega)$ (see [AGI2]).

\section{Gradient estimates}

For a given bounded Lipschitz domain $D \subset \mathbb{R}^{d}, T>0$ and $\Omega=D \times(0, T)$ define the parabolic distance of $(x, t) \in \Omega$ to the parabolic boundary of $\Omega, \partial_{\text {par }} \Omega=$ $(D \times\{0\}) \cup(\partial D \times[0, T))$, by $\delta(x, t)=\inf \left\{\rho((x, t) ;(y, s)):(y, s) \in \partial_{\text {par }} \Omega\right\}$. Here $\rho$ is the standard parabolic distance $\rho((x, t) ;(y, s))=\max \{|x-y|, \sqrt{|t-s|}\}$ in $\mathbb{R}^{d+1}$.

Let us first introduce the two basic maximal functions involved in the pointwise estimate that can be proved following closely the lines of the proof of Corollary 5.2 in [AGI1]. The one-sided Hardy-Littlewood maximal operator on $(0, T)$ is given by

$$
M^{-} g(t)=\sup _{0<h<t} \frac{1}{h} \int_{t-h}^{t}|g(s)| d s
$$

for $t \in(0, T)$, when $g$ is a locally integrable function on $(0, T)$. On the other hand, for a given smooth function $f$ on $D$, the local version of the Calderón maximal function of order $\lambda$ is given at a point $x \in D$, by

$$
M_{D}^{\#, \lambda} f(x)=\sup _{0<r<\tilde{\delta}(x)} \frac{1}{|B(x, r)|^{1+\frac{\lambda}{d}}} \int_{B(x, r)}\left|f(y)-P_{x}(y)\right| d y
$$

where $\tilde{\delta}(x)=\inf \{|x-y|: y \in \partial D\}$ and $P_{x}$ is the Taylor polynomial of degree $\ell-1$ for $f$ at $x$, with $\ell$ the smallest integer larger than $\lambda$. With the above notation and the arguments in the proof of Corollary 5.2 in [AGI1] we get the following result.

Theorem 4. There exists a constant $C$ such that for each $u \in \Theta(\Omega)$ the inequality

$$
\delta^{\ell-\lambda}\left|\nabla^{\ell} u\right| \leq C M^{-}\left[M_{D}^{\#, \lambda} u\right]
$$

holds in $\Omega$, where $\nabla^{\ell} u$ denotes the vector of all the space derivatives of $u$ of order $\ell$.

From the above result we shall obtain the $L^{p}$ estimates which shall be used in the proof of Theorem 1 . We denote by $L_{p}\left((0, T) ; B_{p}^{\lambda}(D)\right)$ the space of all measurable functions $v(x, t)$ on $\Omega$ such that the norm

$$
\|v\|_{L_{p}\left((0, T) ; B_{p}^{\lambda}(D)\right)}=\left(\int_{0}^{T}\|v(\cdot, t)\|_{B_{p}^{\lambda}(D)}^{p} d t\right)^{\frac{1}{p}}
$$

is finite. 
Corollary 5. For some constant $C$ and every $u \in \Theta(\Omega)$, we have the inequality

$$
\left\|\delta^{\ell-\lambda}\left|\nabla^{\ell} u\right|\right\|_{L_{p}(\Omega)} \leq C\|u\|_{L_{p}\left((0, T) ; B_{p}^{\lambda}(D)\right)} .
$$

The proof of Corollary 5 follows from the $L_{p}(0, T)$ boundedness of $M^{-}$and the boundedness of $M_{D}^{\#, \lambda}$ as an operator from $B_{p}^{\lambda}(D)$ to $L_{p}(D)$ (see [DS]).

For the proof of Theorem 2, we shall make use of the next result which is contained in Theorem 1.1 in [AGI2] and remarks following it.

Theorem 6. For $\gamma>0,1<q<\infty$ and $0<\varepsilon<\gamma$ we have that

$$
\Theta(\Omega) \cap L_{q}\left((0, T) ; B_{q}^{\gamma}(D)\right) \subset \mathbb{B}_{q}^{\gamma-\varepsilon}(\Omega) .
$$

\section{Partition of the Wavelet index Set at time $t$}

As before we consider the functions $\delta(x, t)$ defined in $\Omega$ and $\tilde{\delta}(x)$ defined in $D$, the parabolic and elliptic distance functions to the parabolic and elliptic boundaries of $\Omega$ and $D$ respectively. It is not difficult to prove that $\delta(x, t)=\min \{\tilde{\delta}(x), \sqrt{t}\}$. This formula allows us to produce the following partition of $\Omega, \Omega^{1}=\{(x, t) \in \Omega$ : $\delta(x, t)=\tilde{\delta}(x)\}$ and $\Omega^{2}$ to be the interior of the set $\{(x, t) \in \Omega: \delta(x, t)=\sqrt{t}\}$.

The above partition of $\Omega$ induces a classification at each time level of the dyadic cubes in the family $\Gamma_{j}=\left\{I \in \mathcal{D}_{j}: Q(I) \cap \bar{D} \neq \varnothing\right\}$. Set $\Gamma_{j, 0}=\left\{I \in \mathcal{D}_{j}: Q(I) \cap \partial D \neq\right.$ $\varnothing\}$ and $\Gamma_{j}^{o}=\Gamma_{j} \backslash \Gamma_{j, 0}$. Now, for the inner cubes $I \in \Gamma_{j}^{o}$ and $0<t<T$, two different position $\Gamma_{j}^{o}{ }^{1}(t)$ and $\Gamma_{j}^{o^{2}}(t)$ of the interval $I$ raised to time $t, I(t)=I \times\{t\}$ are still possible according to the above decomposition of $\Omega$. In Figure 1, some typical possible situations for an interval $I \in \Gamma_{j}$ are sketched; $I_{1} \in \stackrel{o}{\Gamma}_{j}^{1}\left(t_{2}\right), I_{2} \in \stackrel{o}{\Gamma}_{j}^{2}\left(t_{2}\right)$ and $I_{3} \in \stackrel{o}{\Gamma}_{j}^{2}\left(t_{1}\right) \cap \stackrel{o}{\Gamma}_{j}^{1}\left(t_{3}\right)$.

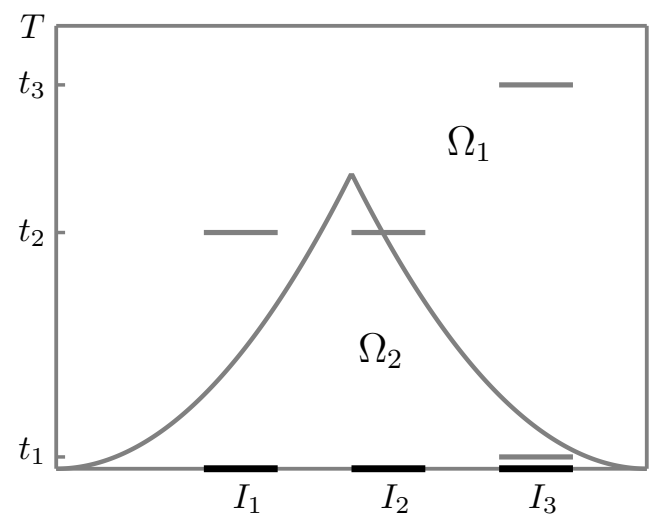

Figure 1. Decomposition of $\Gamma_{j}$.

Precisely, define

$$
\stackrel{o}{\Gamma}_{j}^{1}(t)=\left\{I \in \stackrel{o}{\Gamma_{j}}: I(t) \subset \Omega^{1}\right\} \quad \text { and } \quad \stackrel{o}{\Gamma}_{j}^{2}(t)=\stackrel{o}{\Gamma}_{j} \backslash \stackrel{o}{\Gamma}_{j}^{1}(t)
$$

For each $k \in \mathbb{Z}^{+}$set $\Gamma_{j, k}=\left\{I \in \Gamma_{j}: k 2^{-j} \leq \tilde{\delta}_{Q(I)}<(k+1) 2^{-j}\right\}$, where $\tilde{\delta}_{Q(I)}$ denotes the elliptic Euclidean distance of $Q(I)$ to $\partial D$. With $\Gamma$ we shall denote the family of all cubes in any $\Gamma_{j}$, i.e. $\Gamma=\cup_{j \geq 0} \Gamma_{j}$. Notice also that $\Gamma_{j}=\cup_{k \geq 0} \Gamma_{j, k}$. 
Let us state and prove some lemmas that we shall use in the next section.

Lemma 7. There exist constants $c_{1}$ and $c_{2}$ such that with $t>c_{1} 4^{-j}$ and $I \in \Gamma_{j}{ }^{2}(t)$, we have that $\delta(x, t) \geq c_{2} \sqrt{t}$ for every $x \in Q(I)$.

Proof. Since, once the regularity and support properties of the wavelets are fixed, the cube $Q(I)$ has measure comparable to that of $I$ and $Q(I) \supset I$, there exists a constant $\nu$ such that $Q(I) \subset \nu I$, the dilation of $I$ with respect to its center by a factor $\nu>0$, for every $I \in \mathcal{D}$. On the other hand, since $I \in \stackrel{o}{\Gamma}_{j}^{2}(t), I(t) \cap \Omega^{2} \neq \varnothing$. So that, there exists $z \in I$ such that $(z, t) \in \Omega^{2}$. In other words $\delta(z, t)=\sqrt{t}$. Pick $x \in Q(I)$, then

$$
\begin{aligned}
\sqrt{t}=\delta(z, t) & \leq \rho((z, t) ;(x, t))+\delta(x, t) \\
& =|x-z|+\delta(x, t) \\
& \leq 2 \nu 2^{-j}+\delta(x, t) .
\end{aligned}
$$

Taking $c_{1}=16 \nu^{2}$ and $c_{2}=\frac{1}{2}$ we have the result, since

$$
\begin{aligned}
\sqrt{t} \leq 2 \nu 2^{-j}+\delta(x, t) & <\frac{2 \nu \sqrt{t}}{\sqrt{c_{1}}}+\delta(x, t) \\
& =\frac{\sqrt{t}}{2}+\delta(x, t) .
\end{aligned}
$$

For the intervals in $\Gamma_{j}^{o}(t)$ instead, we have the following result.

Lemma 8. For every $t>0$, every $I \in \Gamma_{j}^{\Gamma^{1}}(t)$ and every $x \in Q(I)$ we have that $\delta(x, t) \geq \widetilde{\delta}_{Q(I)}$

Proof. Since $I \in \Gamma_{j}^{o}{ }^{1}(t)$, then for $x \in Q(I)$ we have that $\delta(x, t)=\tilde{\delta}(x) \geq \tilde{\delta}_{Q(I)}$.

The next lemma contains properties of the families $\Gamma_{j, k}$ which shall be important in the proof of our main result. The second one is a consequence of the regularity of the boundary of $D$, the first and the third of the boundedness of $D$. With \# $E)$ we shall denote the number of elements of the set $E$.

Lemma 9. There exist three constants $C_{0}, C_{1}$ and $C_{2}$ depending on $D$ such that (9.1) $\#\left(\Gamma_{j}\right) \leq C_{0} 2^{j d}$,

(9.2) $\#\left(\Gamma_{j, k}\right) \leq C_{1} 2^{j(d-1)}$ for every $j, k \geq 0$,

(9.3) $\Gamma_{j, k}=\varnothing$ for $k>C_{2} 2^{j}$.

Proof. The estimate (9.1) follows directly from the boundedness of $D$. Notice that (9.3) holds with $C_{2}=\operatorname{diam} D$, since if $I \in \Gamma_{j, k}$ we necessarily have that

$$
k 2^{-j} \leq \tilde{\delta}_{Q(I)} \leq \tilde{\delta}_{I} \leq \operatorname{diam} D
$$

hence $k \leq 2^{j} \operatorname{diam} D$.

Let us briefly sketch the proof of (9.2) for $k=0$. Since $D$ is a bounded Lipschitz domain in $\mathbb{R}^{d}$, we have that the $(d-1)$-Hausdorff measure $\sigma(\partial D)$ of the boundary of $D$ is positive and finite. For $h>0,0<\alpha<\frac{\pi}{2},|\nu|=1, \nu \in \mathbb{R}^{d}$ and $x \in \mathbb{R}^{d}$, set $K(x, h, \alpha, \nu)$ to denote the cone $\{y:|(y-x) \cdot \nu| \geq|x-y| \cos \alpha$ and $|y-x|<h\}$. 
From the Lipschitz character of $\partial D$, there exist a positive number $h$ and $\alpha \in\left(0, \frac{\pi}{2}\right)$ such that for every $x \in \partial D$ there exists a unit vector $\nu(x)$, for which the cone $K(x):=K(x, h, \alpha, \nu(x))$ intersects $\partial D$ only at $x$.

Since $D$ is a bounded domain and the constant $C_{1}$ is expected to depend on $D$, we may assume that $j$ is large enough in order to have $3|Q|^{\frac{1}{d}} 2^{-j}<h$. For each $I \in \Gamma_{j, 0}$ set $\widetilde{Q}(I)$ to denote the cube concentric with $Q(I)$ with side length equal to three times the side length of $Q(I)$. Since $Q(I) \cap \partial D \neq \varnothing$ because $I \in \Gamma_{j, 0}$, we can take a point $x \in Q(I) \cap \partial D$. Now since $(\partial D \cap \widetilde{Q}(I))-\{x\} \subset(K(x))^{c}$, the complementary of $K(x)$, we have that $\sigma(\partial D \cap \widetilde{Q}(I)) \geq \frac{1}{2} \sigma(\partial K(x) \cap \widetilde{Q}(I)) \geq C_{3} 2^{-j(d-1)}$ where $C_{3}$ depends on $\alpha$. Hence $\Gamma_{j, 0} \subset \widetilde{\Gamma}_{j, 0}=\left\{I \in \Gamma_{j}: \sigma(\widetilde{Q}(I) \cap \partial D) \geq C_{3} 2^{-j(d-1)}\right\}$, and it is enough to obtain an estimate for $\#\left(\widetilde{\Gamma}_{j, 0}\right)$ of the form (9.2). Since the overlapping of the family $\left\{\widetilde{Q}(I): I \in \Gamma_{j}\right\}$ is bounded by a constant $M$ which is independent of $j$, we have that

$$
\begin{aligned}
\sigma(\partial D) & =\int_{\mathbb{R}^{d}} \mathcal{X}_{\partial D}(y) d \sigma(y) \\
& \geq \frac{1}{M} \int_{\mathbb{R}^{d}}\left(\sum_{I \in \widetilde{\Gamma}_{j, 0}} \mathcal{X}_{\partial D \cap \widetilde{Q}(I)}(y)\right) d \sigma(y) \\
& =\sum_{I \in \widetilde{\Gamma}_{j, 0}} \sigma(\partial D \cap \widetilde{Q}(I)) \\
& \geq C_{3} \#\left(\widetilde{\Gamma}_{j, 0}\right) 2^{-j(d-1)} .
\end{aligned}
$$

\section{Proof of the Results}

Proof of Theorem 2. Once we have Theorem 1, Theorem 2 follows readily from Theorem 6 in $\S 3$. In fact, since $\mathbb{B}_{p}^{\lambda}(\Omega)=\left(L_{p}(\Omega), W_{p}^{2,1}(\Omega)\right)_{\frac{\lambda}{2}, p}, L_{p}(\Omega)=L_{p}\left((0, T) ; L_{p}(D)\right)$ and since $W_{p}^{2,1}(\Omega)$ is a Banach subspace of $L_{p}\left((0, T) ; W_{p}^{2}(D)\right)$, with $W_{p}^{2}(D)$ the usual elliptic Sobolev space on $D$, a classical result by Lions and Peetre (see $[\mathrm{BL}])$ allows us to claim that $\mathbb{B}_{p}^{\lambda}(\Omega) \subseteq L_{p}\left((0, T) ; B_{p}^{\lambda}(D)\right)$. Hence $\Theta(\Omega) \cap \mathbb{B}_{p}^{\lambda}(\Omega) \subset$ $\Theta(\Omega) \cap L_{p}\left((0, T) ; B_{p}^{\lambda}(D)\right)$.

From Theorem 1, we have that $\Theta(\Omega) \cap \mathbb{B}_{p}^{\lambda}(\Omega) \subseteq L_{\tau}\left((0, T) ; B_{\tau}^{\alpha}(D)\right)$. Now, since $\alpha<d\left(1-\frac{1}{p}\right)$, then $\tau>1$ and Theorem 6 holds with $q=\tau$ and $\gamma=\alpha$. So that $\Theta(\Omega) \cap \mathbb{B}_{p}^{\lambda}(\Omega) \subset \mathbb{B}_{\tau}^{\alpha-\varepsilon}(\Omega)$ for every $\varepsilon>0$, which proves the result.

Proof of Theorem 1. Let us start by taking a temperature $u=u(x, t)$ in the space $L_{p}\left((0, T) ; B_{p}^{\lambda}(D)\right)$ with $1<p<\infty$ and $\lambda>0$. We have to check that $u$ belongs to $L_{\tau}\left((0, T) ; B_{\tau}^{\alpha}(D)\right)$ with $\frac{1}{\tau}=\frac{1}{p}+\frac{\alpha}{d}, 0<\alpha<\min \left\{\ell, \frac{\lambda d}{d-1}\right\}$ and $\ell$ the largest integer less than $\lambda+d$.

For $0<t<T$, set $U(t)$ to denote the function defined in $D$ by $U(t)(x)=u(x ; t)$. Since $u \in L_{p}\left((0, T) ; B_{p}^{\lambda}(D)\right)$, then there exists a subset $Z \subset(0, T)$ of zero measure such that for every $t \notin Z, U(t) \in B_{p}^{\lambda}(D)$. For such a $t$, since $D$ is a Lipschitz 
domain, $U(t)$ can be extended to the whole $\mathbb{R}^{d}$ as a $B_{p}^{\lambda}\left(\mathbb{R}^{d}\right)$ function $V(t)$ with $\|V(t)\|_{B_{p}^{\lambda}\left(\mathbb{R}^{d}\right)} \leq C\|U(t)\|_{B_{p}^{\lambda}(D)}$.

Set $v(x, t)=V(t)(x)$ for $t \notin Z$ and $x \in \mathbb{R}^{d}$ and $v(x, t)=0$ when $t \in Z$. Hence, $\int_{0}^{T}\|V(t)\|_{B_{p}^{\lambda}\left(\mathbb{R}^{d}\right)}^{p} d t \leq C \int_{0}^{T}\|U(t)\|_{B_{p}^{\lambda}(D)}^{p} d t$ and $v \in L_{p}\left((0, T) ; B_{p}^{\lambda}\left(\mathbb{R}^{d}\right)\right)$. Notice that if we apply at each $t \notin Z$ Stein's extension operator [Ste] (see also [JK]) to $U(t)$ to obtain $V(t)$ we get a $v(x ; t)=V(t)(x)$ which is measurable as a function of the variables $(x ; t) \in \mathbb{R}^{d+1}$.

From (2.1) and for fixed $t \in(0, T)$ we have that

$$
\begin{aligned}
V(t) & =P_{0} V(t)+\sum_{I \in \mathcal{D}^{+}} \sum_{\psi \in \Psi}\left\langle V(t), \psi_{I, p^{\prime}}\right\rangle \psi_{I, p} \\
& =\sum_{I \in \mathcal{D}_{0}}\left\langle V(t), \varphi_{I}\right\rangle \varphi_{I}+\sum_{I \in \mathcal{D}^{+}} \sum_{\psi \in \Psi}\left\langle V(t), \psi_{I, p^{\prime}}\right\rangle \psi_{I, p} .
\end{aligned}
$$

in the $B_{p}^{\lambda}\left(\mathbb{R}^{d}\right)$ norm.

We can obtain another $B_{p}^{\lambda}\left(\mathbb{R}^{d}\right)$ function $W(t)$ by restriction of the above sums to those cubes $I$ for which $Q(I)$ intersects $D$. Precisely, we define

$$
W(t)=\sum_{I \in \Gamma_{0}}\left\langle V(t), \varphi_{I}\right\rangle \varphi_{I}+\sum_{I \in \Gamma} \sum_{\psi \in \Psi}\left\langle V(t), \psi_{I, p^{\prime}}\right\rangle \psi_{I, p}=W_{0}(t)+W_{1}(t)
$$

which certainly belongs to $B_{p}^{\lambda}\left(\mathbb{R}^{d}\right)$ and $W(t)=V(t)=U(t)$ on $D$. To prove that $u \in L_{\tau}\left((0, T) ; B_{\tau}^{\alpha}(D)\right)$, it is enough to show that $\int_{0}^{T}\|W(t)\|_{B_{\tau}^{\alpha}\left(\mathbb{R}^{d}\right)}^{\tau}$ is finite.

Let us start by estimating $\int_{0}^{T}\left\|W_{0}(t)\right\|_{B_{\tau}^{\alpha}\left(\mathbb{R}^{d}\right)}^{\tau} d t$. Notice that for each $t \in(0, T)$ $W_{0}(t)$ is a linear combination of a finite number of the $\varphi_{I}$ 's. From Hölder's inequality,

$$
\begin{aligned}
\left\|W_{0}(t)\right\|_{B_{\tau}^{\alpha}\left(\mathbb{R}^{d}\right)} & \leq \sum_{I \in \Gamma_{0}}\left|\left\langle V(t), \varphi_{I}\right\rangle\right|\left\|\varphi_{I}\right\|_{B_{\tau}^{\alpha}\left(\mathbb{R}^{d}\right)} \\
& \leq\|V(t)\|_{L_{p}\left(\mathbb{R}^{d}\right)} \sum_{I \in \Gamma_{0}}\left\|\varphi_{I}\right\|_{L_{p^{\prime}}\left(\mathbb{R}^{d}\right)}\left\|\varphi_{I}\right\|_{B_{\tau}^{\alpha}\left(\mathbb{R}^{d}\right)} \\
& \leq C\|V(t)\|_{B_{p}^{\lambda}\left(\mathbb{R}^{d}\right)} \\
& \leq C\|U(t)\|_{B_{p}^{\lambda}(D)} .
\end{aligned}
$$

Hence

$$
\int_{0}^{T}\left\|W_{0}(t)\right\|_{B_{\tau}^{\alpha}\left(\mathbb{R}^{d}\right)}^{\tau} d t \leq C \int_{0}^{T}\|U(t)\|_{B_{p}^{\lambda}(D)}^{\tau} d t .
$$

Since $\tau<p$, applying Hölder inequality with $\frac{p}{\tau}$ we obtain that

$$
\int_{0}^{T}\left\|W_{0}(t)\right\|_{B_{\tau}^{\alpha}\left(\mathbb{R}^{d}\right)}^{\tau} d t \leq C T^{\frac{p-\tau}{p}}\left(\int_{0}^{T}\|U(t)\|_{B_{p}^{\lambda}(D)}^{p} d t\right)^{\frac{\tau}{p}}
$$

which is finite since $u \in L_{p}\left((0, T) ; B_{p}^{\lambda}(D)\right)$.

The difficult estimate, as in the elliptic case considered in [DD], is actually the proof of the finiteness of $\int_{0}^{T}\left\|W_{1}(t)\right\|_{B_{\tau}^{\alpha}\left(\mathbb{R}^{d}\right)}^{\tau} d t$, where the characterization of $B_{\tau}^{\alpha}\left(\mathbb{R}^{d}\right)$ given by (2.3) in (B) of Proposition 3 becomes crucial. Applying (2.3) 
with $f=W_{1}$, we observe that it suffices to prove that

$$
\int_{0}^{T} \sum_{I \in \Gamma} \sum_{\psi \in \Psi}\left|\left\langle V(t), \psi_{I, p^{\prime}}\right\rangle\right|^{\tau} d t<\infty
$$

From now on we shall use the notation and the lemmas proved in $\S 4$. Hence, with $c_{1}$ the constant in Lemma 7 , we can write the above integral as

$$
\begin{aligned}
\int_{0}^{T} \sum_{I \in \Gamma} \sum_{\psi \in \Psi}\left|\left\langle V(t), \psi_{I, p^{\prime}}\right\rangle\right|^{\tau} d t & =\sum_{j=0}^{\infty} \int_{0}^{T} \sum_{I \in \Gamma_{j}} \sum_{\psi \in \Psi}\left|\left\langle V(t), \psi_{I, p^{\prime}}\right\rangle\right|^{\tau} d t \\
= & \sum_{j=0}^{\infty} \int_{0}^{c_{1} 4^{-j}} \sum_{I \in \Gamma_{j}} \sum_{\psi \in \Psi}\left|\left\langle V(t), \psi_{I, p^{\prime}}\right\rangle\right|^{\tau} d t \\
& +\sum_{j=0}^{\infty} \int_{c_{1} 4^{-j}}^{T} \sum_{I \in \Gamma_{j}} \sum_{\psi \in \Psi}\left|\left\langle V(t), \psi_{I, p^{\prime}}\right\rangle\right|^{\tau} d t \\
= & A+B .
\end{aligned}
$$

Now, since for fixed $j \geq 0$ and $t \in(0, T)$ each $\Gamma_{j}$ is the disjoint union of $\Gamma_{j, 0}, \stackrel{o}{\Gamma_{j}^{1}}(t)$ and $\stackrel{o}{\Gamma}_{j}^{2}(t)$ we have that

$$
\begin{aligned}
B= & \sum_{j=0}^{\infty} \int_{c_{1} 4^{-j}}^{T} \sum_{I \in \Gamma_{j, 0}} \sum_{\psi \in \Psi}\left|\left\langle V(t), \psi_{I, p^{\prime}}\right\rangle\right|^{\tau} d t \\
& +\sum_{j=0}^{\infty} \int_{c_{1} 4^{-j}}^{T} \sum_{\substack{o^{1} \\
I \in \Gamma_{j} \\
(t)}} \sum_{\psi \in \Psi}\left|\left\langle V(t), \psi_{I, p^{\prime}}\right\rangle\right|^{\tau} d t \\
& +\sum_{j=0}^{\infty} \int_{c_{1} 4^{-j}}^{T} \sum_{\substack{o^{2}{ }^{2} \\
\Gamma_{j}(t)}} \sum_{\psi \in \Psi}\left|\left\langle V(t), \psi_{I, p^{\prime}}\right\rangle\right|^{\tau} d t \\
= & B_{0}+B_{1}+B_{2} .
\end{aligned}
$$

So that we have to show that, under the assumption in the statement of Theorem 1 for $d, \lambda, p, \alpha$ and $\tau, A, B_{0}, B_{1}$ and $B_{2}$ are all finite.

Estimate of $A$. It follows from Lemma 9 and (2.2) in Propositon 3 after an adequate application of Hölder's inequality. In fact since

$$
A=\sum_{I \in \Gamma} \sum_{\psi \in \Psi} \int_{0}^{T} \mathcal{X}_{\left(0, c_{1} 4^{-j}\right)}(t) 2^{-j \lambda \tau}\left(2^{j \lambda}\left|\left\langle V(t), \psi_{I, p^{\prime}}\right\rangle\right|\right)^{\tau} d t,
$$

we can apply Hölder's inequality with $\frac{p}{\tau}>1$, the measure on $\Gamma \times \Psi \times(0, T)$ obtained as product of the counting measures on $\Gamma$ and $\Psi$, times the Lebesgue length on $(0, T)$ and the functions $f(I, \psi, t)=\left(2^{j(I) \lambda}\left|\left\langle V(t), \psi_{I, p^{\prime}}\right\rangle\right|\right)^{\tau}$ and $g(I, \psi, t)=$ $\mathcal{X}_{\left(0, c_{1} 4^{-j}\right)}(t) 2^{-j(I) \lambda \tau}$, where $j(I)$ is the resolution level of $I \in \Gamma$, in other words 
$I \in \Gamma_{j(I)}$. Hence $A$ is bounded above by

$$
\begin{aligned}
& \left(\sum_{\Gamma} \sum_{\Psi} \int_{0}^{T} 2^{j(I) p \lambda}\left|\left\langle V(t), \psi_{I, p^{\prime}}\right\rangle\right|^{p} d t\right)^{\frac{\tau}{p}} \cdot\left(\sum_{j} \sum_{\Gamma_{j}} \sum_{\Psi} \int_{0}^{c_{1} 4^{-j}} 2^{-j(I) \lambda \tau} \frac{p}{p-\tau} d t\right)^{\frac{p-\tau}{p}} \\
& \leq\left(\int_{0}^{T} \sum_{\mathcal{D}^{+}} \sum_{\Psi} 2^{j(I) p \lambda}\left|\left\langle V(t), \psi_{I, p^{\prime}}\right\rangle\right|^{p} d t\right)^{\frac{\tau}{p}} \cdot C\left(\sum_{j=0}^{\infty} 2^{-j\left(\tau \lambda \frac{p}{p-\tau}+2-d\right)}\right)^{\frac{p-\tau}{p}} \\
& \leq C\left(\int_{0}^{T}\|V(t)\|_{B_{p}^{\lambda}\left(\mathbb{R}^{d}\right)}^{p} d t\right)^{\frac{\tau}{p}} \\
& \leq C\left(\int_{0}^{T}\|U(t)\|_{B_{p}^{\lambda}(D)}^{p} d t\right)^{\frac{\tau}{p}}, \\
& \text { since } \tau \lambda \frac{p}{p-\tau}+2-d>0 \text { because } 0<\alpha<\frac{\lambda d}{d-1}<\frac{\lambda d}{d-2} .
\end{aligned}
$$

Estimate of $B_{0}$. The series $B_{0}$ can be estimated as $A$ by using Hölder's inequality. The basic difference is that, even when now the time integral is computed on a large interval, the families $\Gamma_{j, 0}$ are smaller than the corresponding families $\Gamma_{j}$ as Lemma 9 shows. In fact, from Hölder's inequality and (9.2),

$$
\begin{aligned}
B_{0} & =\sum_{j=0}^{\infty} \int_{c_{1} 4^{-j}}^{T}\left(\sum_{I \in \Gamma_{j, 0}} \sum_{\psi \in \Psi}\left|\left\langle V(t), \psi_{I, p^{\prime}}\right\rangle\right|^{\tau}\right) d t \\
& \leq \sum_{j} \sum_{\Gamma_{j, 0}} \sum_{\Psi} \int_{0}^{T} 2^{-j \lambda \tau}\left(2^{j \lambda}\left|\left\langle V(t), \psi_{I, p^{\prime}}\right\rangle\right|\right)^{\tau} d t \\
& \leq C\left(\int_{0}^{T} \sum_{j} \sum_{\Gamma_{j, 0}} \sum_{\Psi} 2^{j \lambda p}\left|\left\langle V(t), \psi_{I, p^{\prime}}\right\rangle\right|^{p} d t\right)^{\frac{\tau}{p}} \cdot\left(\sum_{j} 2^{-j \lambda \tau} \frac{p}{p-\tau} 2^{j(d-1)}\right)^{\frac{p-\tau}{p}} \\
& \leq C\left(\int_{0}^{T}\|U(t)\|_{B_{p}^{\lambda}(D)}^{p} d t\right)^{\frac{\tau}{p}},
\end{aligned}
$$

since, being $0<\alpha<\frac{d \lambda}{d-1}$, the exponent $\lambda \tau \frac{p}{p-\tau}-d+1$ of $2^{-j}$ in the last geometric series is positive.

Estimate of $B_{1}$. Now we have to estimate

$$
B_{1}=\sum_{j=0}^{\infty} \int_{c_{1} 4^{-j}}^{T} \sum_{\substack{o 1 \\ I \in \Gamma_{j}(t)}} \sum_{\psi \in \Psi}\left|\left\langle V(t), \psi_{I, p^{\prime}}\right\rangle\right|^{\tau} d t,
$$

where $\stackrel{o}{\Gamma_{j}^{1}}(t)=\left\{I \in \stackrel{o}{\Gamma_{j}}: I(t) \subset \Omega^{1}\right\}$ and $\Omega^{1}=\{(x, t) \in \Omega: \delta(x, t)=\tilde{\delta}(x)\}$.

The next result contains an upper bound for $\left|\left\langle V(t), \psi_{I, p^{\prime}}\right\rangle\right|$ which shall be used to estimate $B_{1}$. 
Claim. There exists a constant $C$ such that

$$
\left|\left\langle V(t), \psi_{I, p^{\prime}}\right\rangle\right| \leq C|Q(I)|^{\frac{\ell}{d}}\left(\int_{Q(I)}\left|\nabla^{\ell} V(t)(x)\right|^{p} d x\right)^{\frac{1}{p}} .
$$

To prove it we shall use the following Poincaré type inequality on each $Q(I)$ for $I \in \mathcal{D}^{+}$,

$$
\left\|V(t)-P_{t, I}\right\|_{L_{p}(Q(I))} \leq C|Q(I)|^{\frac{\ell}{d}}\left(\int_{Q(I)}\left|\nabla^{\ell} V(t)(x)\right|^{p} d x\right)^{\frac{1}{p}},
$$

where, for each $t$ and each $I \in \mathcal{D}^{+}, P_{t, I}(x)$ is a polynomial of degree less than $\ell$ in the variable $x$. Since each $\psi_{I, p^{\prime}}$ has zero moments up to the order $n \geq \ell-1$, and $L_{p^{\prime}}$ norm independent of $I$, applying Hölder and Poincaré inequalities we obtain the claim,

$$
\begin{aligned}
\left|\left\langle V(t), \psi_{I, p^{\prime}}\right\rangle\right| & \leq\left|\left\langle V(t)-P_{t, I}, \psi_{I, p^{\prime}}\right\rangle\right| \\
& \leq\left\|V(t)-P_{t, I}\right\|_{L_{p}(Q(I))}\left\|\psi_{I, p^{\prime}}\right\|_{L_{p^{\prime}}\left(\mathbb{R}^{d}\right)} \\
& \leq C|Q(I)|^{\frac{\ell}{d}}\left(\int_{Q(I)}\left|\nabla^{\ell} V(t)(x)\right|^{p} d x\right)^{\frac{1}{p}} .
\end{aligned}
$$

From (5.1) when $I \in \Gamma_{j}^{o 1}(t)$, Lemma 8 gives

$$
\left|\left\langle V(t), \psi_{I, p^{\prime}}\right\rangle\right| \leq C 2^{-j \ell} \widetilde{\delta}_{Q(I)}^{(\lambda-\ell)}\left(\int_{Q(I)}\left|\delta^{\ell-\lambda}(x, t)\right| \nabla^{\ell} V(t)(x)||^{p} d x\right)^{\frac{1}{p}}
$$

Hence

$$
\begin{aligned}
& B_{1} \leq C \sum_{j} \int_{0}^{T} \sum_{\substack{o \\
\Gamma_{j}}} 2^{-j \ell \tau} \widetilde{\delta}_{Q(I)}^{(\lambda-\ell) \tau}\left(\int_{Q(I)}\left|\delta^{\ell-\lambda}(x, t)\right| \nabla^{\ell} V(t)(x)||^{p} d x\right)^{\frac{\tau}{p}} \\
& \leq C \sum_{j}\left[\sum_{\substack{o \\
\Gamma_{j}}}\left(2^{-j \ell \tau} \widetilde{\delta}_{Q(I)}^{(\lambda-\ell) \tau}\right)^{\frac{p}{p-\tau}}\right]^{\frac{p-\tau}{p}} \cdot\left[\int_{0}^{T} \sum_{\substack{o \\
\Gamma_{j}}} \int_{Q(I)}\left|\delta^{\ell-\lambda}(x, t)\right| \nabla^{\ell} V(t)(x)||^{p} d x\right]^{\frac{\tau}{p}} \\
& \leq C\left(\sum_{j}\left[\sum_{\substack{o \\
\Gamma_{j}}}\left(2^{-j \ell \tau} \widetilde{\delta}_{Q(I)}^{(\lambda-\ell) \tau}\right)^{\frac{p}{p-\tau}}\right]^{\frac{p-\tau}{p}}\right) \cdot\left(\int_{0}^{T} \int_{D}\left|\delta^{\ell-\lambda}(x, t)\right| \nabla^{\ell} V(t)(x)||^{p} d x\right)^{\frac{\tau}{p}}
\end{aligned}
$$

where in the first inequality we have used (5.2), in the second Hölder's inequality for the integral in $t$ and the sum in $I$ for $j$ fixed with the exponents $\frac{p}{\tau}$ and $\frac{p}{p-\tau}$, and in the last one we have used that for fixed $j$ the cubes $Q(I) \subset D$ and their overlapping is uniformly bounded.

Since on $D$ we have that $V(t)=U(t)$, from Corollary 5 we see that the second term on the right hand side of $(5.3)$ is bounded above by a constant times 
$\|u\|_{L_{p}\left((0, T) ; B_{p}^{\lambda}(D)\right)}^{\tau}$. Hence to finish the estimate of $B_{1}$ all we have to do is to prove that the series $\sum_{j}\left(\sum_{\Gamma_{j}}\left(2^{-j \ell \tau} \widetilde{\delta}_{Q(I)}^{(\lambda-\ell) \tau}\right)^{\frac{p}{p-\tau}}\right)^{\frac{p-\tau}{p}}$ converges. Since $\stackrel{o}{\Gamma_{j}}=\cup_{k \geq 1} \Gamma_{j, k}$, from Lemma 9, we get

$$
\begin{aligned}
\left(\sum_{I \in \Gamma_{j}^{o}}\left(2^{-j \ell \tau} \widetilde{\delta}_{Q(I)}^{(\lambda-\ell) \tau}\right)^{\frac{p}{p-\tau}}\right)^{\frac{p-\tau}{p}} & =\left(\sum_{k=1}^{C 2^{j}} \sum_{I \in \Gamma_{j, k}}\left(2^{-j \ell \tau} \widetilde{\delta}_{Q(I)}^{(\lambda-\ell) \tau}\right)^{\frac{p}{p-\tau}}\right)^{\frac{p-\tau}{p}} \\
& \leq C\left(\sum_{k=1}^{C 2^{j}} 2^{j(d-1)} 2^{-\frac{j \ell \tau p}{p-\tau}}\left(k 2^{-j}\right)^{\frac{(\lambda-\ell) \tau p}{p-\tau}}\right)^{\frac{p-\tau}{p}} \\
& =C\left(2^{j\left(d-1-\frac{\lambda \tau p}{p-\tau}\right)} \sum_{k=1}^{C 2^{j}} \frac{(\lambda-\ell) \tau p}{p-\tau}\right)^{\frac{p-\tau}{p}} \\
& \leq C\left(2^{j\left(d-1-\frac{\lambda \tau p}{p-\tau}+\frac{(\lambda-\ell) \tau p}{p-\tau}+1\right)}\right)^{\frac{p-\tau}{p}} \\
& =C 2^{j\left(\frac{d(p-\tau)}{p}-\lambda \tau+(\lambda-\ell) \tau\right)} .
\end{aligned}
$$

Now, since $\frac{d(p-\tau)}{p}-\lambda \tau+(\lambda-\ell) \tau$ is negative the series converge.

Estimate of $B_{2}$. Even when the general scheme for the boundedness of $B_{2}$ looks similar to that for $B_{1}$, we would like to emphasize that they are quite different. This is reflected by the fact that for $B_{2}, t>c_{1} 4^{-j}$ is relevant to apply Lemma 7 to the intervals in $\stackrel{o}{\Gamma}_{j}^{2}(t)$. Since (5.1) holds for each cube $I$ in $\mathcal{D}^{+}$, appling Lemma 7 we get

$$
\begin{aligned}
B_{2} & \leq \sum_{j=0}^{\infty} \int_{c_{1} 4^{-j}}^{T}\left(\sum_{\substack{o^{2} \\
I \in \Gamma_{j}(t)}} \sum_{\psi \in \Psi}\left|\left\langle V(t), \psi_{I, p^{\prime}}\right\rangle\right|^{\tau}\right) d t \\
& \leq C \sum_{j=0}^{\infty} \int_{c_{1} 4^{-j}}^{T} \sum_{\substack{\text { o }^{2} \\
I \in \Gamma_{j}(t)}}|Q(I)|^{\frac{\ell \tau}{d}}\left(\int_{Q(I)}\left|\nabla^{\ell} V(t)(x)\right|^{p} d x\right)^{\frac{\tau}{p}} d t \\
& \leq C \sum_{j=0}^{\infty} \int_{c_{1} 4^{-j}}^{T} \sum_{\substack{\mathcal{O}^{2} \\
I \in \Gamma_{j}(t)}} 2^{-j \ell \tau} t^{\frac{(\lambda-\ell) \tau}{2}}\left(\int_{Q(I)}\left(\delta^{\ell-\lambda}(x, t)\left|\nabla^{\ell} V(t)(x)\right|\right)^{p} d x\right)^{\frac{\tau}{p}} d t .
\end{aligned}
$$


Applying Hölder's inequality for the integral in $t$ and the sum in $I$, for fixed $j$, with exponents $\frac{p}{\tau}$ and $\frac{p}{p-\tau}$, we obtain

$$
B_{2} \leq C\|u\|_{L_{p}\left((0, T) ; B_{p}^{\lambda}(D)\right)}^{\tau} \sum_{j=0}^{\infty}\left(\int_{c_{1} 4^{-j}}^{T} \sum_{\substack{o^{2} \\ I \in \Gamma_{j}(t)}}\left(2^{-j \ell \tau} t^{\frac{(\lambda-\ell) \tau}{2}}\right)^{\frac{p}{p-\tau}} d t\right)^{\frac{p-\tau}{p}} .
$$

Hence, to finish the proof of the theorem, all we have to do is to show that the series

$$
\sum_{j=0}^{\infty}\left(\int_{c_{1} 4^{-j}}^{T} \sum_{\substack{o^{2} \\ I \in \Gamma_{j}^{\prime}(t)}}\left(2^{-j \ell \tau} t^{\frac{(\lambda-\ell) \tau}{2}}\right)^{\frac{p}{p-\tau}} d t\right)^{\frac{p-\tau}{p}}=: S
$$

is finite,

$$
\begin{aligned}
S & =\sum_{j=0}^{\infty} 2^{-j \ell \tau}\left(\int_{c_{1} 4^{-j}}^{T} \sum_{\substack{I_{0} \\
\Gamma_{j}}} \mathcal{X}_{\Gamma_{j}{ }^{2}(t)}(I) t^{\frac{(\lambda-\ell) \tau p}{2(p-\tau)}} d t\right)^{\frac{p-\tau}{p}} \\
& \leq \sum_{j=0}^{\infty} 2^{-j \ell \tau}\left(\sum_{k \geq 1} \int_{c_{1} 4^{-j}}^{T} \sum_{I \in \Gamma_{j, k}} \mathcal{X}_{\Gamma_{j}^{2}(t)}(I) t^{\frac{(\lambda-\ell) \tau p}{2(p-\tau)}} d t\right)^{\frac{p-\tau}{p}} .
\end{aligned}
$$

Notice now that if $I \in \Gamma_{j, k}$ and $t>\left(k 2^{-j}\right)^{2}$, we have that $\sqrt{t}>\widetilde{\delta}(Q(I))$, so that $I \in \stackrel{o}{\Gamma}_{j}^{1}(t)$. Hence in the last estimate obtained for $S$, the indicator function $\mathcal{X}_{\Gamma_{j}^{2}(t)}^{{ }_{{ }^{2}}}(I)$ is bounded above by $\mathcal{X}_{\left(0,\left(k 2^{-j}\right)^{2}\right)}(t)$. Then

$$
\begin{aligned}
S & \leq \sum_{j=0}^{\infty} 2^{-j \ell \tau}\left(\sum_{k \geq \sqrt{c_{1}}} \sum_{I \in \Gamma_{j, k}} \int_{c_{1} 4^{-j}}^{\left(k 2^{-j}\right)^{2}} t^{\frac{(\lambda-\ell) \tau p}{2(p-\tau)}} d t\right)^{\frac{p-\tau}{p}} \\
& \leq C \sum_{j=0}^{\infty} 2^{-j \ell \tau} 2^{-2 j\left[\frac{(\lambda-\ell) \tau}{2}+\frac{p-\tau}{p}\right]}\left(\sum_{k \geq \sqrt{c_{1}}} \sum_{I \in \Gamma_{j, k}}\left(k^{2\left[\frac{(\lambda-\ell) \tau p}{2(p-\tau)}+1\right]}-c_{1}^{\left[\frac{(\lambda-\ell) \tau p}{2(p-\tau)}+1\right]}\right)\right)^{\frac{p-\tau}{p}} \\
& \leq C \sum_{j=0}^{\infty} 2^{-j \ell \tau} 2^{-2 j\left[\frac{(\lambda-\ell) \tau}{2}+\frac{p-\tau}{p}\right]} 2^{j(d-1) \frac{p-\tau}{p}}\left(\sum_{k \geq 1} k^{2\left[\frac{(\lambda-\ell) \tau p}{2(p-\tau)}+1\right]}\right)^{\frac{p-\tau}{p}} \\
& \leq C \sum_{j=0}^{\infty} 2^{-j \ell \tau} 2^{-2 j\left[\frac{(\lambda-\ell) \tau}{2}+\frac{p-\tau}{p}\right]} 2^{j(d-1) \frac{p-\tau}{p}} 2^{j \frac{p-\tau}{p}} 2^{2 j\left[\frac{(\lambda-\ell) \tau}{2}+\frac{p-\tau}{p}\right]} \\
& =C \sum_{j=0}^{\infty} 2^{-j\left(\ell \tau-\frac{d(p-\tau)}{p}\right)},
\end{aligned}
$$

which is finite since $\ell \tau-\frac{d(p-\tau)}{p}<0$. 


\section{REFERENCES}

[AGI1] Hugo Aimar, Ivana Gómez, and Bibiana Iaffei, Parabolic mean values and maximal estimates for gradients of temperatures, J. Funct. Anal. 255 (2008), no. 8, 1939-1956. MR 2462582

[AGI2] Hugo Aimar, Ivana Gómez, and Bibiana Iaffei, On Besov regularity of temperatures, J. Fourier Anal. Appl. 16 (2010), no. 6, 1007-1020.

[BIN] Oleg V. Besov, Valentin P. Il'in, and Sergey M. Nikol'skiı̌, Integral representations of functions and imbedding theorems. Vol. I+II, V. H. Winston \& Sons, Washington, D.C., 1978, 1979, Scripta Series in Mathematics, Edited by Mitchell H. Taibleson. MR 521808 (80f:46030b)

[Bow] Marcin Bownik, The construction of $r$-regular wavelets for arbitrary dilations, J. Fourier Anal. Appl. 7 (2001), no. 5, 489-506. MR 1845100 (2002i:42044)

[DD] Stephan Dahlke and Ronald A. DeVore, Besov regularity for elliptic boundary value problems, Comm. Partial Differential Equations 22 (1997), no. 1-2, 1-16. MR 97k:35047

[DS] Ronald A. DeVore and Robert C. Sharpley, Maximal functions measuring smoothness, Mem. Amer. Math. Soc. 47 (1984), no. 293, viii+115. MR 85g:46039

[FJ] Michael Frazier and Björn Jawerth, A discrete transform and decompositions of distribution spaces, J. Funct. Anal. 93 (1990), no. 1, 34-170. MR 1070037 (92a:46042)

[JK] David Jerison and Carlos E. Kenig, The inhomogeneous Dirichlet problem in Lipschitz domains, J. Funct. Anal. 130 (1995), no. 1, 161-219. MR 96b:35042

[Lei] Christopher Leisner, Nonlinear wavelet approximation in anisotropic Besov spaces, Indiana Univ. Math. J. 52 (2003), no. 2, 437-455. MR 1976084 (2004c:42069)

[Mey] Yves Meyer, Wavelets and operators, Cambridge Studies in Advanced Mathematics, vol. 37, Cambridge University Press, Cambridge, 1992, Translated from the 1990 French original by D. H. Salinger. MR 1228209 (94f:42001)

[Pee] Jaak Peetre, New thoughts on Besov spaces, Duke University Mathematics Series, vol. 1, Mathematics Department, Duke University, Durham, N.C., 1976. MR 57 \#1108

[Sch] Hans-Jürgen Schmeisser, Anisotropic spaces. II. Equivalent norms for abstract spaces, function spaces with weights of Sobolev-Besov type, Math. Nachr. 79 (1977), 55-73. MR 0467286 (57 \#7148)

[ST] Hans-Jürgen Schmeisser and Hans Triebel, Anisotropic spaces. I. Interpolation of abstract spaces and function spaces, Math. Nachr. 73 (1976), 107-123. MR 0430814 (55 \#3819)

[Ste] Elias M. Stein, Singular integrals and differentiability properties of functions, Princeton Mathematical Series, No. 30, Princeton University Press, Princeton, N.J., 1970. MR 44 \#7280 